\title{
Role of State-to-State Kinetics in Determining Transport Coefficients for Hypersonic Flow Simulations
}

\author{
Eswar Josyula ${ }^{*}, 1$, Jonathan M. Burt ${ }^{1}$, William F. Bailey ${ }^{2}$ and Prakash Vedula ${ }^{3}$ \\ ${ }^{1}$ Air Force Research Laboratory, Wright-Patterson AFB, Ohio 45433, USA \\ ${ }^{2}$ Air Force Institute of Technology, Wright-Patterson AFB, Ohio 45433, USA \\ ${ }^{3}$ University of Oklahoma, Norman, Oklahoma 73019, USA
}

\begin{abstract}
A numerical study is performed to assess the influence of thermochemical nonequilibrium on the transport coefficients used in Computational Fluid Dynamics (CFD) simulations of hypersonic external flowfields. An quantitative assessment is made of transport coefficients from simplified methods of Blottner curve fits and Variable Hard Sphere (VHS) model on the numerical solution of a Mach 23 flow past a sphere cone, the RAMC-II test case. The equations derived by Kustova [1] in the state kinetic approach for calculating transport coefficients from the Chapman-Enskog solution of the Wang-Chang Uhlenbeck equation were used to conduct a parametric study for assessment of the effect of the following parameters on the state-specific diffusion coefficients: (1) Widely different population distributions, (2) Atomic mass concentration, and (3) Binary atomic to molecular diffusion coefficient ratio. The present study is a first step to quantify the relative importance of the parameters considered for a future implementation of the computationally expensive state-kinetic transport coefficients in multi-dimensional fluid dynamic flow solvers for flow conditions where the more general state kinetic approach becomes necessary.
\end{abstract}

Keywords: Hypersonic flows, state-to-state kinetics, transport coefficients.

\section{INTRODUCTION}

The design of vehicles for high speed flight, in particular the thermal protection system (TPS) in the highly collisional aerothermal environment, requires development of reliable nonequilibrium models for Computational Fluid Dynamics (CFD) solvers. The limitations, development, and use of current and future CFD models for species transport of mass, momentum, and energy with sources and sinks requires a thorough understanding of the physics of nonequilibrium. The three so called transport properties of a fluid are the coefficients of diffusion, viscosity, and thermal conductivity $[2,3]$. Diffusion is the mass transport through molecular exchange and occurs in a fluid because of random molecular motion. In a macroscopic sense, mass diffusion in a fluid flow occurs due to concentration gradients. In a microscopic sense, the transport of molecules through otherwise identical molecules as a result of concentration gradient is known as self-diffusion. With the idea of momentum transport, viscosity can be stated as the property of the fluid which relates applied stress to the strain rate, where the coefficient of viscosity is directly related to molecular interactions and thus may be considered a thermodynamic property. Thermal conductivity is associated with the transport of mean thermal energy in the flow, where this mean energy consists of random translational energy and internal energy of the molecules.

*Address correspondence to this author at the Air Force Research Laboratory, Wright-Patterson AFB, Ohio 45433, USA;

Tel: 937-713-7100; Fax: 937-656-7867;

E-mail: Eswar.Josyula@us.af.mil
The Navier-Stokes equations are generally applicable in the study of gaseous systems that are close to the equilibrium state. A primary assumption in dealing with polyatomic and reacting gases is that the volumetric rate terms are negligible compared with those associated with spatial gradients. This implies that the inelastic collisions (those associated with vibrational energy exchange or dissociation) are relatively infrequent and do not significantly influence the transport coefficients. In recent years, however, vibrational excitation and chemical reactions are treated as source terms in fluid dynamic codes, where the sources and sinks necessitate modifications of the equations of motion appropriately. In the absence of information on the cross-sections involved, the use of Blottner curve fits [4] for viscosity, Eucken's relation [5] for thermal conductivity and suitable mixing rules are often used in CFD codes, whereas a variable hard sphere (VHS) model [6] in DSMC provide good estimates for transport properties in air. However, with knowledge of the collision cross section, it is convenient to use the Chapman-Enskog approximation [3] to obtain the transport coefficients for use in hypersonic CFD codes. The ChapmanEnskog approximation is used to find the solution of WangChang Uhlenbeck equation [7], the transport equation for the phase space distribution function, which includes terms for internal energy exchanges in the collision integral. Considerable work has been carried out on development and tabulation of a database for the collision integral, appearing in the Sonine polynomial expansion of the Boltzmann equation for computing transport coefficients [8-10] for aerthermodynamic analysis; the limitation of the work is the inability to distinguish the different quantum energy states. 
This limitation is overcome in the approach given in the works of $[1,11-13]$ that includes the vibrational energy states in the collision operator of the transport equation, thus providing state to state transport coefficients for calculation of the transport properties in a gas flow. A justification for this more general state kinetic approach for transport coefficients for given sets of flow conditions was provided in earlier work, see for example $[14,15]$.

Although the nonequilibrium effects are inherent to the transport coefficients of Blottner curve fits, which were derived from experimental data, there is no explicit treatment of the different thermal energy modes or the degree of nonequilibrium in deriving them, and this raises questions on the limits of applicability of these curve fits to nonequilibrium flow processes. There is also uncertainty in the limits of applicability of the VHS model, which is based on a power law molecular potential, for flows involving strong vibrational nonequilibrium. In the present study, a quantitative assessment is made of the relative differences in transport coefficients calculated from Blottner curve fits [4] and the VHS model [6] in air mixture of a high temperature hypersonic flow. Then, using the state kinetic approach of Kustova [1], the role of binary and self diffusion is identified in a gas mixture.

\section{GOVERNING EQUATIONS}

Consider a multi-species, reacting gas mixture, whose underlying distribution functions corresponding to each species $c$, (quantized) vibrational energy level $i$ and (quantized) rotational energy level $j$ are represented as $f_{c i j}\left(x, u_{c}, t\right)$, where $x, u_{c}$ and $t$ denote the spatial coordinate, (microscopic) velocity and time instance respectively. The evolution of these distribution functions, based on kinetic theory, can be expressed as [1,7,11-13]

$\partial_{t} f_{c i j}+u_{c} \cdot \partial_{x} f_{c i j}=J_{c i j}$,

where $J_{c i j}$ denotes the full collision operator that accounts for interactions not only due to elastic collisions but also due to rotational and vibrational energy exchanges and chemical reactions. While each of these mechanisms can be associated with a characteristic time scale, it was found from experimental data (under certain conditions) that there exists a separation of time scales according to the relation $\tau_{e l}<\tau_{\text {rot }}<<\tau_{v i b}<\tau_{\text {react }} \sim \theta$, where $\tau_{e l}, \tau_{\text {rot }}, \tau_{\text {vib }}, \tau_{\text {react }}$ and $\theta$ correspond to mean times characterizing collisions resulting in translational, rotational, vibrational energy transfer, chemical reactions and macroscopic time scale respectively. In other words, as translational and rotational energy modes tend to equilibrate much faster in comparison to other modes, the collision operator on the r.h.s of Eq. (1) can be expressed as $J_{c i j}=\frac{1}{\varepsilon} J_{c i j}^{\text {rapid }}+J_{c i j}^{\text {slow }}$, where $J_{c i j}^{\text {rapid }} \equiv J_{c i j}^{e l}+J_{c i j}^{\text {rot }} \quad$ and $\quad J_{c i j}^{\text {slow }} \equiv J_{c i j}^{v i b}+J_{c i j}^{\text {react }} \quad$ correspond to collision operators of rapid and slow processes and $\varepsilon$ is a small parameter denoting the ratio of timescales of fast and slow (collisional) processes. Note that the collision operators
$J_{c i j}^{e l}, J_{c i j}^{r o t}, J_{c i j}^{v i b}$ and $J_{c i j}^{\text {react }}$ correspond to elastic collisions, rotational energy exchanges, vibrational energy exchanges and chemical reactions, respectively. While a direct solution of Eq. (1) can be very computationally expensive, the generalized Chapman-Enskog method can be used to obtain approximate solutions. In this approach, the distribution functions $f_{c i j}$ are expressed in a power series involving the (small) parameter $\varepsilon$. The zeroth order solution $f_{c i j}^{(0)}$ satisfies the relation $J_{c i j}^{e l}\left(f_{c i j}^{(0)}, f_{c i j}^{(0)}\right)+J_{c i j}^{r o t}\left(f_{c i j}^{(0)}, f_{c i j}^{(0)}\right)=0$. Consideration of collision invariants, leads to the following form for the zeroth order distribution function:

$f_{c i j}^{(0)}=\left(\frac{m_{c}}{2 \pi k T}\right)^{3 / 2} s_{j}^{c i} \frac{n_{c i}}{Z_{c i}^{r o t}(T)} \exp \left(-\frac{m_{c} c_{c}^{2}}{2 k T}-\frac{\varepsilon_{j}^{c i}}{k T}\right)$,

where $n_{c i}$ is the number density of molecules of species $c$ (with molecular mass $m_{c}$ ) in vibrational level $i, s_{j}^{c i}$ denotes the statistical weight, $k$ is the Boltzmann constant, $T$ is the temperature, $c_{c} \equiv u_{c}-v$ is the peculiar velocity, $\mathbf{v}$ is the macroscopic gas velocity and $Z_{c i}^{\text {rot }}$ is the rotational partition function. The macroscopic parameters $n_{c i}(x, t), v(x, t)$ and $T(x, t)$ are defined in terms of the distribution function and satisfy the following relations:

$$
\begin{aligned}
& n_{c i}=\sum_{j} \int f_{c i j} d \mathbf{u}_{c}=\sum_{j} \int f_{c i j}^{(0)} d \mathbf{u}_{c} \\
& \rho v=\sum_{c i j} m_{c} \int \mathbf{u}_{c} f_{c i j} d \mathbf{u}_{c}=\sum_{c i j} m_{c} \int \mathbf{u}_{c} f_{c i j}^{(0)} d \mathbf{u}_{c} \\
& \rho U=\sum_{c i j} \int\left(\frac{1}{2} m_{c} c_{c}^{2}+\varepsilon_{i}^{c}+\varepsilon_{j}^{c i}+\varepsilon^{c}\right) f_{c i j} d \mathbf{u}_{c} \\
& =\sum_{c i j} \int\left(\frac{1}{2} m_{c} c_{c}^{2}+\varepsilon_{i}^{c}+\varepsilon_{j}^{c i}+\varepsilon^{c}\right) f_{c i j}^{(0)} d \mathbf{u}_{c} .
\end{aligned}
$$

Note that $U$ denotes the total energy per unit mass defined such that $\rho U \equiv \frac{3}{2} n k T+\rho E_{r}+\rho E_{v}+\rho E_{f}$. For each species $c$ and vibrational level $i$, the corresponding (mass) density is defined as $\rho_{c i}=m_{c} n_{c i}$, the vibrational energy of the molecule is given by $\varepsilon_{i}^{c}$ (based on reference minimum of its potential curve, $\varepsilon^{c}=-D_{c}$, where $D_{c}$ is the energy of dissociation of the molecular species $c$ ). The components of total energy per unit volume due to rotation, vibration and dissociation are given by $\rho E_{r}=\sum_{c i j} \int \varepsilon_{j}^{c i} f_{c i j} d \mathbf{u}_{c}$, $\rho E_{v}=\sum_{c i} \varepsilon_{i}^{c} n_{c i}$ and $\rho E_{f}=\sum_{c} \varepsilon^{c} n_{c}$ respectively. Also note that the number density of species $c$ is given by $n_{c}=\sum_{i} n_{c i}$, the total number of particles is given by $n=\sum_{c i} n_{c i}$ and the density of the gas mixture is given by $\rho=\sum_{c} m_{c} \sum_{i} n_{c i}$. 
Based on Eqs. (1)-(6), macroscopic equations governing the evolution of $n_{c i}(x, t), v(x, t)$ and $T(x, t)$ for a multicomponent reacting gas mixture in vibrational and chemical nonequilibrium can be obtained as

$\frac{d n_{c i}}{d t}+n_{c i} \nabla \cdot v+\nabla \cdot\left(n_{c i} \mathbf{V}_{c i}\right)=R_{c i}$

$\rho \frac{d v}{d t}+\nabla \cdot \mathbf{P}=0$

$\rho \frac{d U}{d t}+\nabla \cdot \mathbf{q}+\mathbf{P}: \nabla \mathbf{v}=0$

where the production terms are given by $R_{c i} \equiv \sum_{j} \int J_{c i j}^{\text {slow }} d \mathbf{u}_{c}$, the diffusion velocity $\mathbf{V}_{c i}$ of the chemical species $c$ at vibrational level $i$ is given by $n_{c i} \mathbf{V}_{c i} \equiv \sum_{j} \int \mathbf{c}_{c} f_{c i j} d \mathbf{u}_{c}$, the pressure tensor is given by $\mathbf{P} \equiv \sum_{c i j} \int m_{c} \mathbf{c}_{c} \mathbf{c}_{c} f_{c i j} d \mathbf{u}_{c}$ and the heat flux vector is given by $\mathbf{q} \equiv \sum_{c i j} \int\left(m_{c} c_{c}^{2} / 2+\varepsilon_{j}^{c i}+\varepsilon_{i}^{c}+\varepsilon^{c}\right) \mathbf{c}_{c} f_{c i j} d \mathbf{u}_{c}$. Based on the zeroth order distribution function, the corresponding approximations for the diffusion velocity, pressure tensor and heat flux vector can be obtained as $\mathbf{V}_{c i}{ }^{(0)}=0, \mathbf{P}^{(0)}=p \mathbf{I}$ and $\mathbf{q}^{(0)}=0$, where $p$ denotes the pressure and $I$ denotes the unit tensor. First order corrections to these approximations can be obtained from linear integral equations for the first order distribution functions $f_{c i j}^{(1)} \equiv f_{c i j}^{(0)} \varphi_{c i j}$. These linear integral equations result in the following form of the first order distribution function:

$$
f_{c i j}^{(1)}=\frac{f_{c i j}^{(0)}}{n}\left(-\mathbf{A}_{c i j} \cdot \nabla \ln T-\sum_{d k} \mathbf{D}_{c i j}^{d k} \cdot \mathbf{d}_{d k}-\mathbf{B}_{c i j}: \nabla \mathbf{v}-F_{c i j} \nabla \cdot \mathbf{v}-G_{c i j}\right)
$$

The functions $\mathbf{A}_{c i j}, \mathbf{B}_{c i j}, \mathbf{D}_{c i j}^{d k}, F_{c i j}$ and $\mathbf{G}_{c i j}$, which depend on microscopic velocities $u_{c}$ and macroscopic parameters $n_{c i}(x, t), \mathbf{v}(x, t)$ and $T(x, t)$ can be identified as coefficients of the underlying gradient terms in the linear integral equations. Note that the diffusion driving forces for each chemical species $c$ at vibrational level $i$ is given by $\mathbf{d}_{c i}=\nabla\left(n_{c i} / n\right)+\left\{\left(n_{c i} / n\right)-\left(\rho_{c i} / \rho\right)\right\} \nabla \ln p$. The above first order distribution functions can be used to obtain expressions for the diffusion velocity, pressure tensor and total heat flux vector as:

$$
\begin{aligned}
& \mathbf{V}_{c i}=-\sum_{d k} D_{c i d k} \mathbf{d}_{d k}-D_{T c i} \nabla \ln T \\
& \mathbf{P}=\left(p-p_{r e l}\right) \mathbf{I}-2 \mu S-\eta \nabla \cdot \mathbf{v I} \\
& \mathbf{q}=-\lambda^{\prime} \nabla T-p \sum_{c i} D_{T c i} \mathbf{d}_{c i}+\sum_{c i}\left(\frac{5}{2} k T+\left\langle\varepsilon_{j}^{c i}\right\rangle_{r}+\varepsilon_{i}^{c}+\varepsilon^{c}\right) n_{c i} \mathbf{V}_{c i} .
\end{aligned}
$$

Here $D_{\text {cidk }}$ and $D_{T c i}$ denote the diffusion coefficient and thermal diffusion coefficient for each chemical and vibrational species, given in terms of bracket integrals as $D_{\text {cidk }}=\frac{1}{3 n}\left[\mathbf{D}^{c i}, \mathbf{D}^{d k}\right]$ and $D_{T c i}=\frac{1}{3 n}\left[\mathbf{D}^{c i}, \mathbf{A}\right]$. Note that the pressure tensor (based on the first order approximation) depends on the strain rate tensor $S$, the shear viscosity coefficient $\mu=\frac{k T}{10}[\mathbf{B}, \mathbf{B}]$, the bulk viscosity coefficient $\eta=k T[F, F]$ and the relaxation pressure $p_{r e l}=k T[F, G]$. As a result of inelastic translational and rotational energy transfers in collisions between molecules of different vibrational and chemical species, additional terms appear in the pressure tensor in the form of relaxation pressure and bulk viscosity. The coefficient of thermal conductivity $\lambda^{\prime}=\frac{k}{3}[\mathbf{A}, \mathbf{A}]$ appearing in the total heat flux vector also depends on the elastic and inelastic exchanges between translational and rotational modes. The transport coefficients corresponding to shear viscosity, thermal conductivity, mass diffusion and thermal diffusion coefficients can be obtained using Sonine and Waldmann-Trubenbacher orthogonal polynomials. An important aspect of this approach for obtaining the transport coefficients is that detailed interactions due to vibrational energy transfers and chemical kinetics are carefully considered and could result in more accurate expressions for diffusion velocity, stress tensor and heat flux vector in the presence of thermochemical nonequilibrium.

Simplifications to the above approach are possible when the diffusion velocity is assumed to be given by a Fick's law as

$\rho_{c i} \mathbf{V}_{c i}=-\rho_{c} D_{11} \nabla\left(\rho_{c i} / \rho_{c}\right)$,

where $D_{11}$ is the self-diffision coefficient which can be obtained from kinetic theory as $D_{11}=\eta^{\prime} \mu / \rho$, where $\eta^{\prime}$ is 1.2 for hard sphere molecules, is 1.43 for Maxwell molecules and is somewhere in between these two values based on experimental results. The species diffusion velocity $\mathbf{V}_{c} \equiv \widetilde{\mathbf{C}}_{c}-\mathbf{v}$ defined as the species mass-averaged velocity $\widetilde{\mathbf{C}}_{c} \equiv \frac{1}{\rho_{c}} \sum_{i, j} m_{c} \int \mathbf{u}_{c} f_{c i j} d \mathbf{u}_{c}$ relative to the mixture massaveraged velocity $\mathbf{v}$, can also be approximated by Fick's law as

$\rho_{c} \mathbf{V}_{c}=-\rho D_{12} \nabla\left(\rho_{c} / \rho\right)$

where the binary diffusion coefficient $D_{12}$ is obtained by assuming a constant Lewis number. These simplified approximations for transport coefficients can be used in conjunction with the global conservation equations as:

$$
\begin{aligned}
& \partial_{t} \rho_{c i}+\nabla \cdot\left[\rho_{c i}\left(\mathbf{v}+\mathbf{V}_{c}+\mathbf{V}_{c i}\right)\right]=\dot{\omega}_{c i} \\
& \partial_{t} \rho_{c}+\nabla \cdot\left[\rho_{c}\left(\mathbf{v}+\mathbf{V}_{c}\right)\right]=\dot{\omega}_{c} \\
& \partial_{t}(\rho \mathbf{v})+\nabla \cdot(\rho \mathbf{v v}+\tilde{\tau})=0
\end{aligned}
$$


$\partial_{t}\left(\rho e_{v i b}\right)+\nabla \cdot\left[\rho e_{v i b}\left(\mathbf{v}+\mathbf{V}_{c}\right)+\dot{\mathbf{q}}_{\text {trans }}+\dot{\mathbf{q}}_{v i b}\right]=\rho \dot{\omega}_{v i b}$

$\partial_{t}(\rho e)+\nabla \cdot\left[\rho(e+p / \rho) v-\sum \dot{\mathbf{q}}_{v i b}+\sum\left(\rho_{c} h_{c} \mathbf{V}_{c}\right)-\mathbf{v} \cdot \tau\right]=0$

The conservation Eq. (16) is used in the state to state kinetics code, written for mass density in quantum level $i$ for diatomic molecules. The source term $\dot{\omega}_{c i}$ derived from the vibrational master equations is made up of the relevant energy exchange processes consisting of the V-T and V-V energy exchange mechanisms. The density of molecular species is the sum of population densities in the various vibrational levels. For diatomic species in the state to state kinetics formulation, a separate vibrational conservation equation was not necessary as the vibrational energy was calculated at each quantum level. The mass conservation of species is represented by Eq. (17). The production of small amounts of atoms due to dissociation of molecules is included in the source term, $\dot{\omega}_{c}$. The mixture density, $\rho$ is the sum of the partial species densities,

$\rho=\rho_{N_{2}}+\rho_{O_{2}}+\rho_{O}+\rho_{N O}+\rho_{N}$

Eq. (18) gives the conservation of total momentum. For diatomic molecules other than those treated with the statekinetic rates, vibrational relaxation in Eq. (19) was modeled according to the Landau-Teller [5-16] form. Eq. (19) also includes terms for the conduction and diffusion of vibrational energy. The conservation of total energy is given by Eq. (20) with heat conduction and species diffusion terms.

\section{RESULTS AND DISCUSSIONS}

Results are presented in two sections. The first section provides comparisons of using two commonly used transport coefficient models for the RAMC-II test case. The second section is a parametric study of binary $\&$ self diffusion on an $N_{2}-N$ gas mixture using a state specific approach.

\subsection{Relative Effects of Simplified Transport Coefficients on RAMC-II Test Case}

In this section, an assessment is made of two simplified transport coefficient models commonly used in current hypersonic CFD codes, based on flow simulation for a RAMC-II test case (Mach 23 air flow at $61 \mathrm{~km}$ altitude). A post-processing technique is used to assess the local influence of the transport coefficient model on diffusion coefficient values throughout the flowfield. The dynamic viscosity for each of the five species in the reacting flow hypersonic sphere-cone simulation is calculated as a function of temperature, using the Blottner model [4] employed in a CFD flow simulation that solved Eqs (17)-(20). This viscosity is given as

$\mu_{\text {Blottner }}=\exp [(A \ln T+B) \ln T+C]$

where $\mathrm{A}, \mathrm{B}$, and $\mathrm{C}$ are species specific constants.

For comparison, species viscosities are also computed for the variable hard sphere (VHS) model of Bird [6] through a series of post-processing calculations based on CFD simulation results. The VHS model is the standard transport coefficient model in the direct simulation Monte Carlo (DSMC) method, and is widely used for a range of gas kinetic schemes due to its ease of implementation and the existence of analytical solutions involving integration over equilibrium velocity distributions. In this model, the cross section for intermolecular collisions scales as a constant power of the relative speed for each collision pair, and the post-collision relative velocity is isotropically distributed. It follows that the viscosity is proportional to a constant power $(\omega)$ of temperature, with the allowable range bounded by a value of $\frac{1}{2}$ for a hard sphere gas and 1 for a Maxwell gas. Given a reference temperature $T_{r e f}$ and a reference collision diameter $d_{r e f}$ at this temperature, the VHS viscosity may be expressed as

$\mu_{V H S}=\frac{15 \sqrt{\pi m k_{B} T_{r e f}}}{2 \pi d_{r e f}^{2}(5-2 \omega)(7-2 \omega)}\left[\frac{T}{T_{r e f}}\right]^{\omega}$

where $m$ is the molecular mass, $T$ is the gas temperature and $k_{B}$ is Boltzmann's constant. As with the Blottner model, the species-specific dynamic viscosity in the VHS model is a function of only temperature and species constants. The local ratio of Blottner viscosity to VHS viscosity may therefore be calculated for each species as a function of temperature. Eucken's relation is employed to calculate species thermal conductivities for both Blottner and VHS models. Species thermal conductivities will therefore have the same dependence on internal energy excitation in both models, and the local ratio of thermal conductivities should equal the corresponding viscosity ratio. In the flow simulation, the mixture viscosity and thermal conductivity values are computed as functions of species transport coefficients and concentrations using the mixing rule of Wilke [5]. As part of post-processing calculations, this mixing rule is applied separately to the species-specific VHS viscosities given by Eq. (23). The resulting local mixture VHS viscosity, $\mu_{V H S}$, mix may then be compared with the mixture viscosity $\mu_{\text {Blottner }}$, mix employed in the simulation by evaluating the ratio $\mu_{\text {Blottner }}$, mix $/ \mu_{V H S}$, mix. Note that, while for individual species, the ratio of Blottner-to-VHS thermal conductivities is equal to the corresponding viscosity ratio, these two ratios are not necessarily equal for the mixture; this follows from the fact that species thermal conductivities depend on the number of internal degrees of freedom, so the relative contributions of each species to summations used in Wilke's mixing rule will be different for thermal conductivity than for viscosity. In order to guarantee that the summation of species diffusive mass fluxes is zero, a single diffusion coefficient is utilized in the simulation through a constant Lewis number approximation. This approximation is equivalent to setting the mixture diffusion coefficient as a function of only the mixture viscosity. The ratio of Blottner to VHS mixture diffusion coefficients is therefore equal to the viscosity ratio $\mu_{\text {Blottner }}$, mix / $\mu_{V H S}$, mix . 
Note that the transport coefficient assessment here is inherently approximate, due in part to the following three factors: First, a separate simulation employing the VHS model is not performed, and instead the same local temperature values are used to compute Blottner and VHS viscosities. Second, in DSMC simulation of a gas mixture, each combination of species involved in a binary collision may be assigned a different reference diameter $d_{\text {ref }}$ and temperature exponent $(\omega)$, which makes application of VHS procedures for a mixture not easily amenable to CFD calculations or analysis. Single species viscosity ratios are used in order to avoid consideration of differences associated with mixing rules, and the Wilke mixing rule is employed as a simplified means of relating VHS and Blottner transport coefficients for a five-species air mixture. Finally, due to the approximate nature of the VHS power law temperature dependence, VHS input parameters are generally only valid over a limited temperature range. The temperature range in the calculated flowfield is considerably wider than the appropriate range for a single set of VHS parameters, so a comparison between VHS and Blottner transport coefficients should be performed with an understanding that VHS coefficient values may not be accurate at all points within the simulation domain.

In order to quantify the variation in transport coefficient ratios among the different species, ratio values extracted along the stagnation streamline are presented in Fig. (1). The corresponding temperature variation is also shown in the figure. We find a maximum value within the highest temperature shock region for $N$, a drop in viscosity ratio values across the shock for all other species, and a relatively small range of ratio values for $N_{2}$ in comparison with the other species. For all species, very little variation in the viscosity ratio is found over the large temperature range (over $10,000 \mathrm{~K}$ ) which characterizes the post-shock region; this trend seems to indicate that the relative validity of the Blottner and VHS models and input parameters may be comparable over this temperature range. For the mixture ratio, it is observed in Fig. (1) that values are greater than one outside a small region around the shock, with a maximum of 1.20 near the outer edge of the thermal boundary layer.

The strong dependence of the mixture Blottner/VHS transport coefficient ratio on nitrogen dissociation and recombination is illustrated in Fig. (2), which shows the variation in mixture ratio along with species mole fractions over the stagnation streamline. In this figure, the maximum ratio value is coincident with the location of highest $N$ concentration, near the outer edge of the boundary layer. Due to comparatively low dissociation energy, $O_{2}$ is shown in

Fig. (2) to almost completely dissociate a short distance downstream of the shock. As a result, the large transport coefficient ratio values for $\mathrm{O}$ (in addition to the large ratio for atomic nitrogen) may be assumed to contribute significantly to a post-shock increase in the mixture ratio. Based on general trends in Fig. (2), including mixture transport coefficient ratio values which are smaller than one near the shock and greater than one elsewhere along the stagnation streamline, we assume that the use of the VHS model in place of the Blottner model would result in a somewhat reduced shock thickness and a slightly thicker boundary layer.

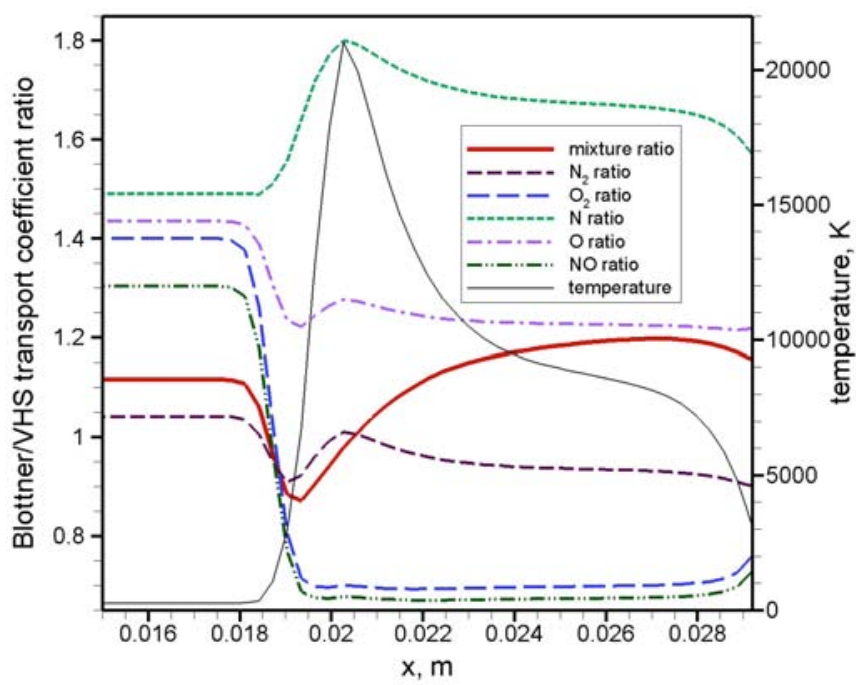

Fig. (1). Variation in transport coefficient ratios and temperature along stagnation streamline, RAMC-II test case at $61 \mathrm{~km}$ altitude, Medium $=$ Air.

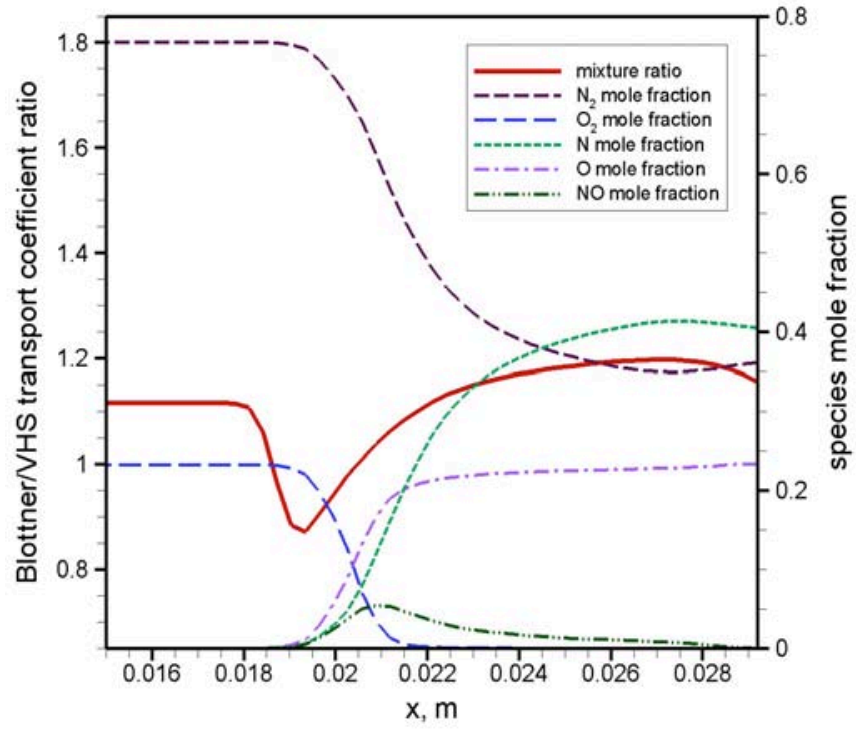

Fig. (2). Mixture transport coefficient ratio and species mole fractions along stagnation streamline, RAMC-II test case at $61 \mathrm{~km}$ altitude, Medium $=$ Air.

Due to the inherent difficulty in adjusting the constants in the Blottner model or VHS parameters for thermochemical nonequilibrium gas mixtures of air, the evaluation of transport coefficients based on state to state kinetic modeling is attractive.

\subsection{Parametric Study of Binary and Self Diffusion in $\mathrm{N}_{2}$ - $N$ Gas Mixture: State Kinetic Approach}

Having assessed the effects of experimental-based and empirical transport models, in this section we assess the importance of state specific transport coefficients for given flow conditions. The work of Kustova [1] lays the foundation for this 
extension. Her formalism is recaptured, recast in a parametric form and then evaluated for a molecular/atomic nitrogen mixture with a focus on vibrational state specific coefficients.

Following Kustova [1] the diffusion coefficients - both binary and self - are expressed in terms of mass fractions of the relevant species. The system under consideration is a mixture of molecular nitrogen and atomic nitrogen. A state specific approach is adopted with the relevant molecular states being the vibrational states. Only a single atomic state is considered. The molecular mass fraction for a given molecular state is recast into a fractional vibrational population form and the diffusion coefficients simplified. The fractional population is displayed in Fig. (3) as a function of the vibrational states. The vibrational temperature, $T_{v}=-\Delta / \ln [n 2 / n 1]$, ranges from 1500 to $3000 \mathrm{~K}$ with $\Delta$ set to $[0.3 \times 11600 \mathrm{~K}]$. An artificially enhanced population in the high vibrational states is introduced (shown in Eq. (24) below) to simulate effects due to anharmonic pumping and biased recombination.

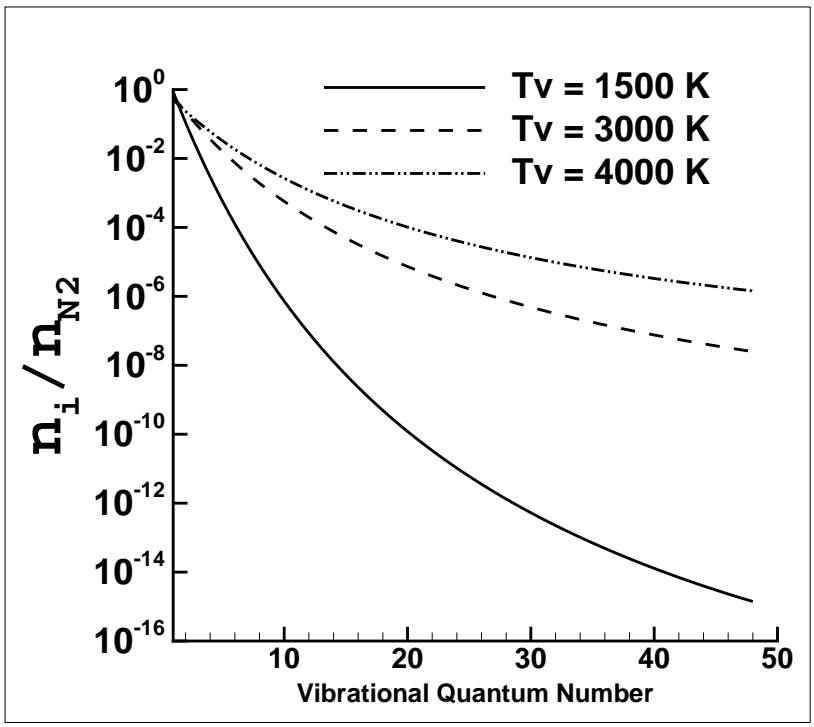

Fig. (3). Fractional population distribution in vibrational energy states from $1500 \mathrm{~K}$ to $4000 \mathrm{~K}$, Medium $=N_{2}-N$ gas mixture.

$n_{i} / n_{N 2}=\exp \left[-0.3 \times i /\left(\left(T_{v} / 11600\right) \times(1+0.0431 \times i)\right)\right]$

$i=0,1,2,3 \ldots 48$

The original form of the self-diffusion coefficient in [1] given below (Eq. (25)) is cast in a functional form that permits parametric studies.

$$
D_{N_{2 i}, N_{2 i}}=\frac{\left(\frac{C_{N}}{\mathcal{D}_{N_{2}}}+\frac{2\left(-1-C_{N}+1 / C_{N_{2 i}}\right)}{\mathcal{D}_{N N_{2}}}\right) \mathcal{D}_{N N_{2}} m_{N}^{2} n^{2}}{\left(\frac{C_{N_{2}}}{2 \mathcal{D}_{N_{2}}}+\frac{C_{N}}{\mathcal{D}_{N N_{2}}}\right) \rho^{2}} i=0,1,2,3 \ldots
$$

This form is achieved by expressing the atomic nitrogen mass fraction as $1 / f$, the ratio of the binary, atomic nitrogen-molecular nitrogen diffusion coefficients $\mathcal{D}_{N_{2}} / \mathcal{D}_{N_{2}}$ as $\beta$ and the vibrational state concentrations, $\xi$, in terms of the fractional populations. The resulting equation,
$D_{N_{2 i}, N_{2 i}}=\mathcal{D}_{N_{2}} \frac{(1+f)^{2} \beta\left[2 f^{2}(-1+\xi)+\xi(-2+\beta)-f \xi \beta\right]}{2(-1+f) f^{2} \xi[2+(-1+f) \beta]}$

asymptotically depends inversely on the fractional population. This is made obvious in Fig. (4) by plotting the product of the state concentration and the state-specific self diffusion coefficient. For this and the future comparisons, we have normalized the diffusion coefficients to the molecular nitrogen self-diffusion coefficient, $\mathcal{D}_{\mathrm{N}_{2}}$. Note that the asymptotic value is insensitive to vibrational temperature and population departures from Boltzmann form.

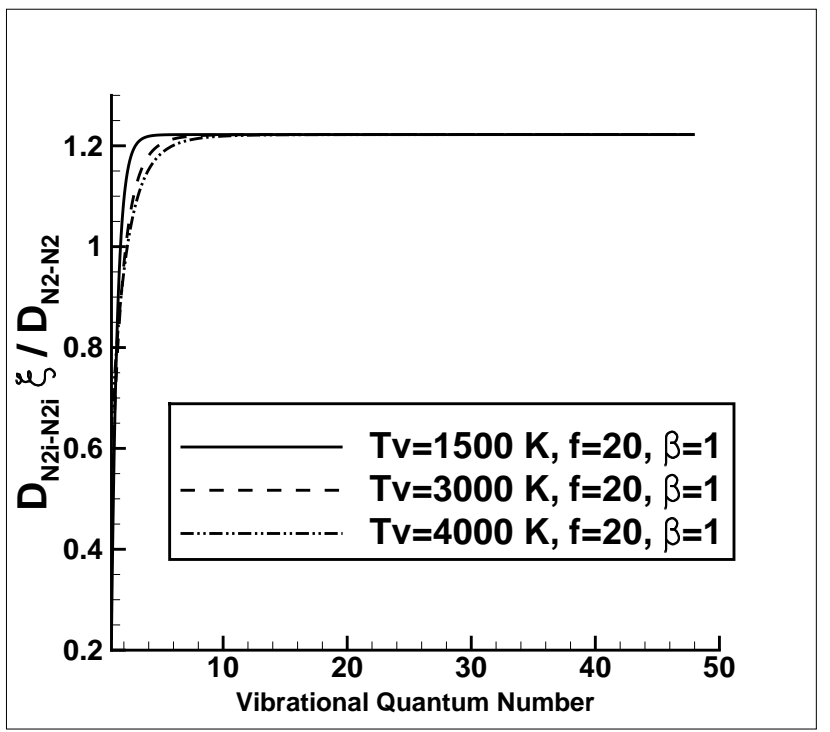

Fig. (4). Self-diffusion coefficient from $1500 \mathrm{~K}$ to $4000 \mathrm{~K}, \mathrm{f}=20$, $\beta=1$, Medium $=N_{2}-N$ gas mixture

$$
D_{N_{2 i} N_{2 k}}=D_{N_{2} N_{2}}=\frac{\left(\frac{C_{N}}{\mathcal{D}_{N_{2}}}-\frac{2\left(1+C_{N}\right)}{\mathcal{D}_{N N_{2}}}\right) \mathcal{D}_{N N_{2}} m_{N}^{2} n^{2}}{\left(\frac{C_{N_{2}}}{2 \mathcal{D}_{N_{2}}}+\frac{C_{N}}{\mathcal{D}_{N N_{2}}}\right) \rho^{2}} i \neq k
$$

is also cast into the parametric representation with the result:

$$
D_{N_{2} N_{2}}=\mathcal{D}_{N_{2}} \frac{(1+f)^{2}(2+2 f-\beta) \beta}{2 f^{2}[2+(-1+f) \beta]}
$$

It is interesting to note that state specific binary diffusion coefficient is directly proportional to the molecular nitrogen binary diffusion coefficient and independent of the states of the collision partners, $\mathrm{i}$ and $\mathrm{k}$.

In Fig. (5), we examine the variation of the binary state specific diffusion coefficient with $f$, the inverse of the atomic mass concentration for $\beta=1$. At small concentrations, less than $20 \%(f>55)$, the coefficient approaches the molecular nitrogen binary diffusion coefficient, $\mathcal{D}_{N_{2}}$. This binary state specific diffusion coefficient is less sensitive to the relative values of the atomic and molecular diffusion coefficients, $\beta$. We examine this dependence in Fig. (6) for $\beta$ ranging from 
0.5 to 2 with $f$ assigned the value of 10 and note less than a $20 \%$ variation.

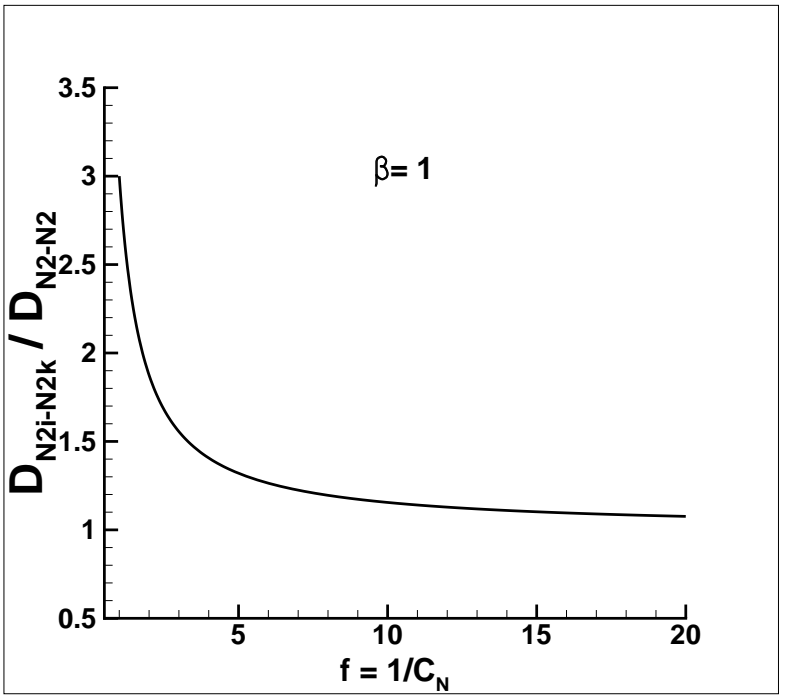

Fig. (5). Variation of binary diffusion coefficient with inverse of mass concentration, $\beta=1$, Medium $=N_{2}-N$ gas mixture.

The state specific vibrational binary diffusion coefficient, $D_{N_{2 i} N_{2 k}}$ given in [1],

In Fig. (7), we examine the sensitivity of the statespecific self-diffusion coefficient to the atomic to molecular ratio, $\beta$ for population distribution at $3000 \mathrm{~K}$ with $f$ set to 10. The dependence is weak over the entire range of $\beta$ from 0.5 to 2.0 , with asymptotic value decreasing as $\beta$ decreases and converging to the state specific binary value. Fixing $\beta$ at 1 and the population distribution at the $3000 \mathrm{~K}$ value, we next examine the variation of the state specific self-diffusion coefficient with respect to atomic concentration, $1 / f$. With $f=2,5$ and 10 , the state specific self-diffusion coefficient decreases monotonically from values near 3 to those near 1 , simultaneously approaching the value of the state specific binary diffusion coefficient, $D_{N_{2 i} N_{2 k}}$, shown earlier in

Fig. (6).

\section{CONCLUDING REMARKS}

A numerical study is performed to assess the influence of thermochemical nonequilibrium on the transport coefficients used in Computational Fluid Dynamics (CFD) simulation of hypersonic flows and to introduce a much more rigorous approach to modeling of the nonequilibrium transport process than the current practice. A quantitative assessment is made of transport coefficients from simplified methods of Blottner curve fits and the Variable Hard Sphere (VHS) model for hypersonic external flowfield of a Mach 23 air flow past a sphere cone, the RAMC-II test case. A parametric study was conducted using a state specific approach to examine the roles of binary and self diffusion in an $N_{2}-N$ gas mixture.

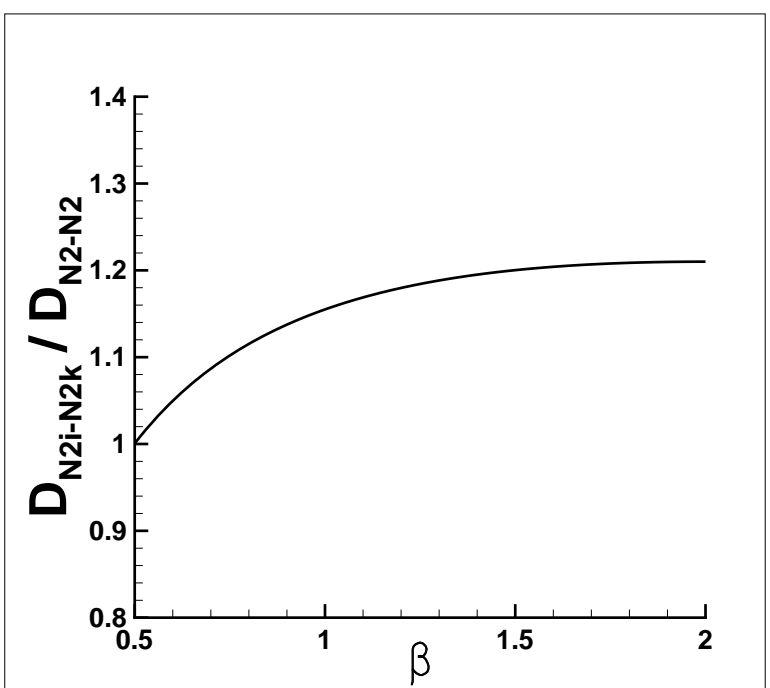

Fig. (6). Variation of binary diffusion coefficient with ratio of atomic to molecular diffusion coefficient $(\beta), f=10$, Medium $=$ $N_{2}-N$ gas mixture.

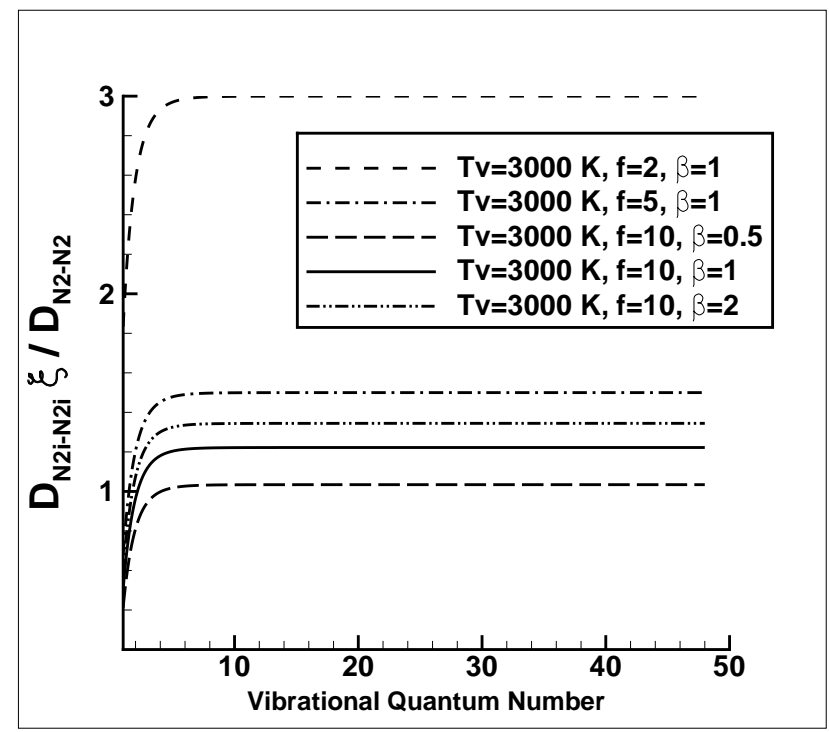

Fig. (7). Variation of self diffusion coefficient in the vibrational energy states at $3000 \mathrm{~K}$, Medium $=N_{2}-N$ gas mixture.

Comparison of simplified transport coefficient models of Blottner curve fits and VHS model for a Mach 23 air flow past a body showed that use of VHS model would result in reduced shock thickness and a slightly thicker boundary layer. Large transport coefficient ratios between Blottner and VHS models for atomic oxygen and nitrogen contribute significantly to a post-shock increase in the Blottner/VHS ratio for a high temperature air mixture. The ratio of viscosity coefficients of the air mixture from the Blottner curve fits and VHS model has a relatively small variation for the large temperature range. However, significant differences in transport coefficients from both models are expected in mixtures with large oxygen concentration. It should be noted that variation in transport coefficient values associated with model selection is a well established fact, and the existence of the discrepancies discussed here is very much expected. The analysis shown in this work is not intended to demonstrate that such discrepancies exist, but instead to quantify the differences among both species-specific and 
mixture transport coefficients for a representative hypersonic flow problem. This analysis shows the potential impact of different models for the temperature dependence of transport coefficients on these coefficients themselves, as well as on predicted flowfield characteristics.

A parametric study of assessing the role of binary and self diffusion in a $N_{2}-N$ gas mixture showed that self diffusion coefficients depend inversely on the population in the quantum energy states. It was found that the asymptotic value for the self diffusion coefficient is insensitive to the vibrational temperature as well as the population distributions, but this value is sensitive to the degree of dissociation. The state specific binary diffusion coefficient is directly proportional to molecular nitrogen binary diffusion coefficient and is independent of the states of the collision partners. For low atomic concentration the state specific binary diffusion coefficient approaches that of diatomic nitrogen. These findings have the potential to significantly impact the implementation and modeling of state-to-state transport coefficients in computational fluid dynamics flow solvers.

\section{CONFLICT OF INTEREST}

The authors confirm that this article content has no conflict of interest.

\section{ACKNOWLEDGEMENTS}

Helpful discussion with E. Kustova of Saint Petersburg University is gratefully acknowledged.

\section{REFERENCES}

[1] Kustova EV. On the simplified state-to-state transport coefficients. Chem Phys 2001; 270: 177-95.

[2] Bird RB, Stewart WE, Lightfoot EN. Transport phenomena. New York: John Wiley \& Sons, Inc 1960

[3] Hirschfelder JO, Curtiss CF, Bird RB. Molecular theory of gases and liquids. New York: John Wiley \& Sons, Inc 1964.

[4] Blottner FG, Johnson M, Ellis M. Chemically reacting viscous flow program for multicomponent gas mixtures. Technical Report: Sandia Laboratories 1971; SC-RR-70-754.

[5] Vincenti WG, Kruger Jr $\mathrm{CH}$. Introduction to physical gas dynamics. New York: John Wiley \& Sons, Inc 1967.

[6] Bird GA. Molecular gas dynamics and the direct simulation of gas flows. Oxford: Clarendon Press 1994.

[7] Ferziger JH, Kaper HG. Mathematical theory of transport processes in gases. New York: North Holland Publishing Compnay 1972.

[8] Palmer G. An assessment of transport property methodologies for hypersonic flows. AIAA Paper pp. 97-0983.

[9] Palmer G, Wright MJ. A comprison of methods to compute high temperature gas thermal conductivity. AIAA Paper 2003: 20033913.

[10] Wright MJ, Bose D, Palmer G, Levin E. Recommended collision integrals for transport property computations, part 1: air species. AIAA J 2005; 43(12): 2558-64.

[11] Kustova EV, Nagnibeda EA. Strong nonequilibrium effects on specific heats and thermal conductivity of diatomic gas. Chem Phys 1996; 208: 313-29.

[12] Kustova EV, Nagnibeda EA. Transport properties of a reacting gas mixture with strong vibrational and chemical nonequilibrium. Chem Phys 1998; 233: 57-75.

[13] Chikhaoui A, Dudon JP, Kustova EV, Nagnibeda EA. Transport properties in reacting mixture of polyatomic gases. Physica A 1997; 247: 526-52.

[14] Bruno D, Capitelli M, Kustova E, Nagnibeda E. Non-equilibrium vibrational distributions and transport coefficients of $\mathrm{N}_{2(\mathrm{v})}-\mathrm{N}$ mixtures. Chem Phys Lett 1999; 308: 463-72.

[15] Orsini A, Rini P, Taviani V, Fletcher D, Kustova EV, Nagnibeda EA. State-to-state simulation of nonequilibrium nitrogen stagnation-line flows: fluid dynamics and vibrational kinetics. J Thermophys Heat Transf 2008; 22: 390-8.

[16] Landau L, Teller E. Zur Theorie der Schalldispersion. Physikalische Zeitschrift der Sowjetunion 1936; 10(1): 34-43. 